\title{
Conformal methods for massless Feynman integrals and large $N_{f}$ methods
}

\author{
J.A. Gracey
}

\begin{abstract}
We review the large $N$ method of calculating high order information on the renormalization group functions in a quantum field theory which is based on conformal integration methods. As an example these techniques are applied to a typical graph contributing to the $\beta$-function of $O(N) \phi^{4}$ theory at $O\left(1 / N^{2}\right)$. The possible future directions for the large $N$ methods are discussed in light of the development of more recent techniques such as the Laporta algorithm.
\end{abstract}

\section{Introduction}

One of the main problems in renormalization theory is the construction of the renormalization group functions. These govern how the parameters of a quantum field theory, such as the coupling constant, depend on scale. In situations where one has to compare with precision data, this ordinarily requires knowing the renormalization group functions to very high orders in a perturbative expansion. The quantum field theories we have in mind are not only the gauge theories of particle physics but also the scalar and fermionic ones which arise in condensed matter problems. These are central in understanding phase transitions. To attain such precision in perturbative expansions means that large numbers of Feynman diagrams have to be determined with the number of graphs increasing with the loop order. Moreover, as the order increases the underlying integrals require more sophisticated methods in order to deduce their value analytically. The widely established methods of computing Feynman graphs will be reported elsewhere in this volume. Here we review an alternative approach which complements explicit perturbative techniques. It does

J.A. Gracey, Theoretical Physics Division, Department of Mathematical Sciences, University of Liverpool, P.O. Box 147, Liverpool, L69 3BX, United Kingdom e-mail: gracey@liv.ac.uk Contribution to book "Computer Algebra in Quantum Field Theory: Integration, Summation and Special Functions" 
so in such a way that for low loop orders there is overlap but at orders beyond that already known part of the perturbative series can be deduced at all orders within a certain approximation. This is known as the large $N$ or large $N_{f}$ method where $N$ is a parameter deriving from a symmetry of the theory such as a Lie group or the number of massless quark flavours, $N_{f}$, in Quantum Chromodynamics (QCD). In this method the Feynman graphs are related to those of perturbation theory but because of the nature of the expansion parameter, the powers of the propagators appearing in such graphs are not the canonical value of unity but instead differ from unity by $O(\varepsilon)$ where $\varepsilon$ corresponds to the regularizing parameter of dimensional regularization. In addition beyond leading order in the $1 / N$ expansion, the propagator powers will include the anomalous dimensions in addition to the leading or canonical dimension. Therefore, standard perturbative techniques such as integration by parts requires care in its use since one may not be able to actually reduce a graph to a simpler topology. Instead a different technique has had to be refined and developed. It is based on a conformal property of Feynman integrals and we review it here in the context of the large $N$ methods. Though it has had some applications in perturbative computations.

The article is organized as follows. We devote the next section to the notation and techniques of computing Feynman graphs using conformal methods in $d$-dimensions. We focus on the general two loop self energy graph in the subsequent section and review the work of [1, 2], upon which this review is mostly based, and others in the methods of evaluating it. These techniques are then applied to a problem in scalar quantum field theory in Section 4 where a graph with 10 internal integrations is evaluated exactly in $d$-dimensions. We conclude in Section 5 with thoughts on the direction in which the technique could be developed next given recent advances in the computation of Feynman graphs using conventional perturbative techniques.

\section{Notation and Elementary Techniques}

We begin by introducing the notation we will use which will be based on [1, 2]. There Feynman graphs were represented in coordinate or configuration space notation. By this we mean that in writing a Feynman integral graphically the integration variables are represented as the vertices. By contrast in momentum space representation the integration variables correspond to the momenta circulating around a loop. So in coordinate space representation propagators are denoted by lines between two fixed points, as illustrated in Figure 1.

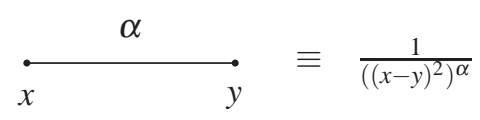

Figure 1. Coordinate space propagator. 
There the power of the propagator is denoted by a number or symbol beside the line. One can map between coordinate and momentum space representation by using a Fourier transform. In the notation of [1, 2] we have

$$
\frac{1}{\left(x^{2}\right)^{\alpha}}=\frac{a(\alpha)}{2^{2 \alpha} \pi^{\mu}} \int_{k} d^{d} k \frac{e^{i k x}}{\left(k^{2}\right)^{\mu-\alpha}}
$$

where $x$ is in coordinate space and $k$ is the conjugate momentum. Also for shorthand we set

$$
d=2 \mu
$$

which is used throughout to avoid the appearance of $d / 2$ in the Euler $\Gamma$-function. This symbol should not be confused with the mass scale appearing in renormalization group equations. Clearly

$$
a(\alpha)=\frac{\Gamma(\mu-\alpha)}{\Gamma(\alpha)}
$$

which is singular when $\alpha=\mu+n$ where $n$ is zero or a positive integer. Also $a(\alpha)$ vanishes at the negative integers. The elementary identity

$$
a(\alpha) a(\mu-\alpha)=1
$$

follows trivially as does

$$
a(\alpha)=\frac{a(\alpha-1)}{(\alpha-1)(\mu-\alpha)}
$$

from the $\Gamma$-function identity $\Gamma(z+1)=z \Gamma(z)$. With this notation the elementary one loop self energy graph in momentum space is replaced by chain integration in coordinate space representation. This is represented graphically in Figure 2

$$
\underset{0}{\stackrel{\alpha}{y} \quad \beta} \equiv v(\alpha, \beta, 2 \mu-\alpha-\beta) \underset{0}{\stackrel{\alpha+\beta-\mu}{x}}
$$

Figure 2. Chain integration.

where, [1, 2],

$$
v(\alpha, \beta, \gamma)=\pi^{\mu} a(\alpha) a(\beta) a(\gamma) .
$$

However, in practice Feynman graphs have more complicated integration points. In other words in coordinate space representation one has more than two lines intersecting at a point. Therefore, more involved integration techniques are required to evaluate the Feynman integrals. One very useful technique is that of uniqueness or conformal integration which was introduced in three dimensions in [3]. It has been developed in several ways subsequently and specifically to $d$-dimensions. For ex- 
ample, see [4]. We follow [1, 2] and use the rule represented in Figure 3, where $z$ is the integration variable,

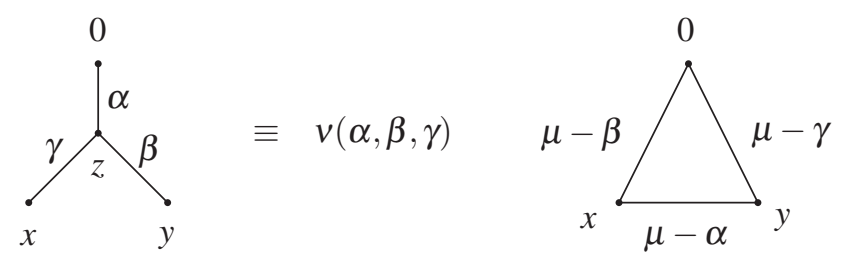

Figure 3. Conformal integration when $\alpha+\beta+\gamma=2 \mu$.

which follows when the sum of the exponents of the lines intersecting at the 3-point vertex add to the spacetime dimension

$$
\alpha+\beta+\gamma=2 \mu .
$$

This is known as the uniqueness condition. By the same token if a graph contains a triangle where the lines comprising the triangle sum to $\mu$ such as

$$
(\mu-\alpha)+(\mu-\beta)+(\mu-\gamma)=\mu
$$

as is the case in Figure 3, then the unique triangle can be replaced by the vertex on the left side. There are several methods to establish the uniqueness integration rule. If one uses standard text book methods such as Feynman parameters then the integral over $z$ can be written as

$$
\begin{aligned}
& \frac{\pi^{\mu} \Gamma(\mu-\alpha) \Gamma(\alpha+\beta+\gamma-\mu)}{\Gamma(\beta) \Gamma(\gamma) \Gamma(\mu)} \\
& \times \int_{0}^{1} d b \frac{b^{\beta-1}(1-b)^{\gamma-1}}{\left[b(1-b)(x-y)^{2}\right]^{\alpha+\beta+\gamma-\mu}} \\
& \times{ }_{2} F_{1}\left(\alpha+\beta+\gamma-\mu, \alpha ; \mu ;-\frac{[b x+(1-b) y]}{b(1-b)(x-y)^{2}}\right)
\end{aligned}
$$

prior to using, (77). When that condition is set then the hypergeometric function collapses to the geometric series and allows the integration over the Feynman parameter $b$ to proceed which results in

$$
\begin{aligned}
& \frac{\pi^{\mu} \Gamma(\mu-\alpha) \Gamma(\mu-\beta) \Gamma(\mu-\gamma)}{\Gamma(2 \mu-\beta-\gamma) \Gamma(\beta) \Gamma(\gamma)\left(y^{2}\right)^{\alpha}\left[(x-y)^{2}\right]^{\beta+\gamma-\mu}} \\
& \times_{2} F_{1}\left(\alpha, \mu-\gamma ; 2 \mu-\beta-\gamma ; 1-\frac{x^{2}}{y^{2}}\right)
\end{aligned}
$$


Applying the uniqueness condition a second time produces the right hand side of Figure 3 since the hypergeometric function again reduces to the geometric series. This is in such a way that the canonical propagators emerge.

An alternative method is to apply a conformal transformation on the coordinates of the integral, [2]. In this approach, which is applicable to any graph in general, one external point is labelled as an origin and given 0 as a coordinate. The other points are denoted by coordinates $x, y$ and $z$. The conformal transformation changes the integration coordinate as well as the external points through

$$
x_{\mu} \rightarrow \frac{x_{\mu}}{x^{2}} .
$$

Thus for two coordinates $y$ and $z$ undergoing such a transformation we have the lemma

$$
(y-z)^{2} \rightarrow \frac{(y-z)^{2}}{y^{2} z^{2}} .
$$

An integration measure also produces contributions to the lines joining to the origin since

$$
d^{d} z \rightarrow \frac{d^{d} z}{\left(z^{2}\right)^{2 \mu}}
$$

Therefore, for the vertex on the left side of Figure 3 this transformation produces the intermediate integral of Figure 4.

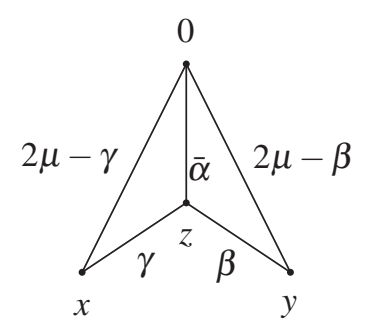

Figure 4. Vertex of Figure 3 after a conformal transformation with base at 0 where

$$
\bar{\alpha}=2 \mu-\alpha-\beta-\gamma \text {. }
$$

To complete the integration requires setting the uniqueness condition (7) which produces a chain integral since the line from 0 to $z$ is absent from the graph. To complete the derivation one undoes the original conformal transformations to produce the right hand side of Figure 3. If one compares the two derivations, the latter is in fact of more practical use. This is because it avoids the use of writing the original integral in terms of Feynman parameters which would become tedious for higher order cases. Also it is simple to implement graphically.

Having recalled the derivation of the uniqueness rule it is straightforward to see that there is a natural extension. In the first derivation there was not a unique way to collapse the hypergeometric function to an elementary type of propagator. Instead 
this will happen if the sum of the exponents is $(2 \mu+n)$ where $n$ is a positive integer. Athough the collapse in this case will not be to the geometric series, it will reduce to simple algebraic functions which are of the propagator type. So, for instance, when $n=1$ we have the result of Figure 5, [5],

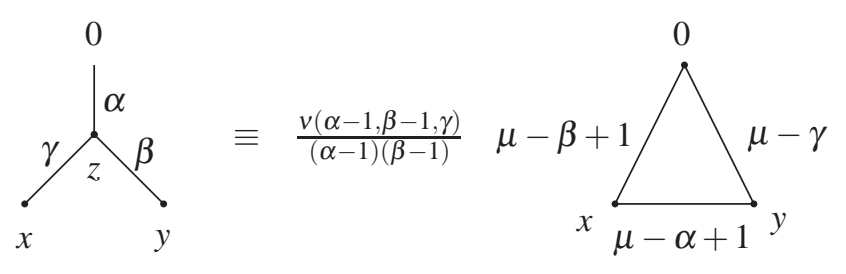

$$
\begin{aligned}
& +\frac{v(\alpha-1, \beta, \gamma-1)}{(\alpha-1)(\gamma-1)} \quad \mu-\beta \sum_{x \sum_{\mu-\alpha+1} y-\gamma+1}^{0} y \\
& +\frac{v(\alpha, \beta-1, \gamma-1)}{(\beta-1)(\gamma-1)} \mu-\beta+1 \bigwedge_{x} \mu-\gamma+1
\end{aligned}
$$

Figure 5. Conformal integration when $\alpha+\beta+\gamma=2 \mu+1$.

A similar rule has been constructed and used in [4]. We will use Figure 4 later in order to simplify various integrals.

\section{Two Loop Self Energy Graph}

We can illustrate some of the techniques of conformal integration by considering the massless two loop self energy graph with arbitrary powers, $\alpha_{i}$ on the propagators. It is illustrated in Figure 6

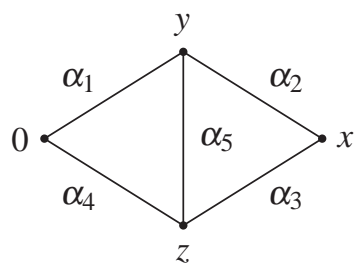

Figure 6. Two loop self energy graph in coordinate space representation. 
where we have used the coordinate space representation. Thus the vertices are integrated over rather than the loop momenta. To clarify, the integral of Figure 6 is

$$
I\left(\alpha_{1}, \alpha_{2}, \alpha_{3}, \alpha_{4}, \alpha_{5}\right)=\int_{y z} \frac{1}{\left(y^{2}\right)^{\alpha_{1}}\left((x-y)^{2}\right)^{\alpha_{2}}\left((x-z)^{2}\right)^{\alpha_{3}}\left(z^{2}\right)^{\alpha_{4}}\left((y-z)^{2}\right)^{\alpha_{5}}}
$$

where $\int_{y}=\int \frac{d^{d} y}{(2 \pi)^{d}}$. The structure of this integral has been widely studied and we briefly highlight several properties of relevance. The analysis of [6, 7] determined that the symmetry group of the graph was $Z_{2} \times S_{6}$ which has 1440 elements. Exploiting this the $\varepsilon$ expansion of the integral in $d=4-2 \varepsilon$ with propagator powers of order $\varepsilon$ from unity was determined up to $O\left(\varepsilon^{6}\right)$, [6, 7]. At $O\left(\varepsilon^{5}\right)$ it was discovered that the first multi-zeta value occurred, [7]. Specifically

$$
\begin{aligned}
I(1,1, \mu-1,1, \mu-1)= & 6 \zeta_{3}+9 \zeta_{4} \varepsilon+7 \zeta_{5} \varepsilon^{5} \\
& +\frac{5}{2}\left[\zeta_{6}-2 \zeta_{3}^{2}\right] \varepsilon^{3}-\frac{1}{8}\left[91 \zeta_{7}+120 \zeta_{3} \zeta_{4}\right] \varepsilon^{4} \\
& +\frac{1}{81920}\left[653440 \zeta_{5} \zeta_{3}-7059417 \zeta_{8}+576 F_{53}\right] \varepsilon^{5} \\
& +O\left(\varepsilon^{6}\right)
\end{aligned}
$$

where $\zeta_{z}$ is the Riemann zeta function and $F_{53}=\sum_{n>m>0} \frac{1}{n^{5} m^{3}}$ in the original notation of [8]. Subsequent to this it has been shown that the only numbers which appear in the full series expansion in $\varepsilon$ are mutiple zeta values, [9]. While the work of [6, 7] illustrated the power of group theory to evaluate master integrals explicitly, using conformal integration allows one to relate two loop self energy integrals by exploiting the masslessness of the original diagrams. This was originally developed in [1, 2] and we summarize that here as there appears to be scope nowadays to take this method to three and higher loop order graphs.

The transformations developed in [2] fall into several classes. The first is that derived from the elementary use of the Fourier transform. Writing

$$
I\left(\alpha_{1}, \alpha_{2}, \alpha_{3}, \alpha_{4}, \alpha_{5}\right)=\frac{\Gamma}{\left(x^{2}\right)^{D-2 \mu}}
$$

where $D=\sum_{i=1}^{5} \alpha_{i}$ and $\Gamma$ is independent of $x$ and corresponds to the value of the integral, then taking the Fourier transform produces an integral which is also the two loop self energy. Though the propagator powers are different. In this sense one can say that the graph is self-dual which is not a property all Feynman graphs have. Thus, [2],

$$
I\left(\alpha_{1}, \alpha_{2}, \alpha_{3}, \alpha_{4}, \alpha_{5}\right)=\frac{\prod_{i=1}^{5} a\left(\alpha_{i}\right)}{a(D-2 \mu)} I\left(\mu-\alpha_{2}, \mu-\alpha_{3}, \mu-\alpha_{4}, \mu-\alpha_{1}, \mu-\alpha_{5}\right) .
$$

This transformation is known as the momentum representation or MR. It can be easily generalized to other topologies and there is a simple graphical rule for this. Although not immediately apparent from the self energy because of the self-duality, 
each 3-valent vertex of the original graph has an associated triangle in the dual graph. For other topologies 4-valent vertices are mapped to squares and 5-valent vertices to pentagons with a clear generalization pattern.

A set of less obvious transformations can be deduced from the uniqueness condition. First, we define the shorthand notation, [2],

$$
\begin{array}{ll}
s_{1}=\alpha_{1}+\alpha_{2}+\alpha_{5} & , \quad s_{2}=\alpha_{3}+\alpha_{4}+\alpha_{5} \\
t_{1}=\alpha_{1}+\alpha_{4}+\alpha_{5} & , \quad t_{2}=\alpha_{2}+\alpha_{3}+\alpha_{5}
\end{array}
$$

and illustrate the technique for one case. If one considers the central propagator it can be replaced by a chain integral. Although there are an infinite number of ways of doing this one can choose the exponents of the chain so that the top vertex is unique. In other words

$$
\begin{aligned}
& \frac{1}{\left((y-z)^{2}\right)^{\alpha_{5}}} \\
& =\frac{1}{v\left(2 \mu-\alpha_{1}-\alpha_{2}, s_{1}-\mu, \mu-\alpha_{5}\right)} \int_{u} \frac{1}{\left((y-u)^{2}\right)^{2 \mu-\alpha_{1}-\alpha_{2}}\left((u-z)^{2}\right)^{s_{1}-\mu}}
\end{aligned}
$$

where $u$ is the intermediate integration point. As the $y$ vertex of Figure 6 is now unique the conformal integration rule can be used to rewrite the integral. This results in, [2],

$$
I\left(\alpha_{1}, \alpha_{2}, \alpha_{3}, \alpha_{4}, \alpha_{5}\right)=\frac{a\left(\alpha_{1}\right) a\left(\alpha_{2}\right) a\left(\alpha_{5}\right)}{a\left(s_{1}-\mu\right)} I\left(\mu-\alpha_{2}, \mu-\alpha_{1}, \alpha_{3}, \alpha_{4}, s_{1}-\mu\right) .
$$

In the notation of [2] this transformation is known as $\uparrow$. It is elementary to see that there are five other such transformations which are denoted by $\nearrow, \nwarrow, \downarrow, \searrow$ and $\swarrow$. The syntax is that when an arrow points in a general upwards direction it is a transformation on the $y$ vertex and by contrast in a downwards direction it relates to the $z$ vertex. The propagator which one replaces by a chain to make the vertex unique is in correspondence with the direction of the arrow. While these six transformations operate on the internal vertices there are two which act on each of the external vertices. One can complete the uniqueness of one of these by realizing that the integral itself is a propagator with power $(D-2 \mu)$ as indicated in [16], [2]. For example, if the right external point is chosen as the base integration vertex then the appending propagator has power $\left(2 \mu-\alpha_{2}-\alpha_{3}\right)$. This produces

$$
I\left(\alpha_{1}, \alpha_{2}, \alpha_{3}, \alpha_{4}, \alpha_{5}\right)=\frac{a\left(\alpha_{2}\right) a\left(\alpha_{3}\right)}{a(D-2 \mu) a\left(2 \mu-t_{1}\right)} I\left(\alpha_{1}, \mu-\alpha_{3}, \mu-\alpha_{2}, \alpha_{4}, t_{2}-\mu\right)
$$

and this is denoted by $\leftarrow$. The corresponding transformation on the left external point is called $\rightarrow$.

The final set of transformations are based on the conformal transformations (11) and (12) together with the effect they have on the two vertex measures, [2]. One can choose either of the external vertices as the origin of the transformation. Once 
decided the result of the conformal transformation is that all propagators joining to the origin have their powers changed to the difference of $2 \mu$ and the sum of the exponents at the point at the other end of that propagator. This means all points including those not directly connected to the base point in the first place. For the two loop self energy there are no such points but for higher loop graphs this will be the case. We will give an example of this in Section 4. Thus the conformal left transformation is, [2],

$$
I\left(\alpha_{1}, \alpha_{2}, \alpha_{3}, \alpha_{4}, \alpha_{5}\right)=I\left(2 \mu-s_{1}, \alpha_{2}, \alpha_{3}, 2 \mu-s_{2}, \alpha_{5}\right)
$$

where there is no $\Gamma$-function factor and this is denoted by $\mathrm{CL}$ in contrast to $\mathrm{CR}$ which is the transformation based on the right external vertex as the origin of the conformal transformation. The full set of transformations and the result of applying each to the graph of Figure 6 are summarized in a Table in [2]. However, as brief examples of the transformations the integral of 15 is related as follows

$$
\begin{aligned}
I(1,1, \mu-1,1, \mu-1) & \stackrel{\uparrow}{=} I(\mu-1, \mu-1,1, \mu-1,1) \\
& \stackrel{\mathrm{CR}}{=} I(\mu-1,1,1,1, \mu-1) .
\end{aligned}
$$

Though the latter follows from a simple rotation of the integral as well.

Aside from the transformations there are other techniques which allow one to evaluate the two loop self energy and higher order graphs. Perhaps the most exploited is that of integration by parts which was introduced for [16) in [10]. It determined that the first term in the $\varepsilon$ expansion of $I(1,1,1,1,1)$ was $6 \zeta_{3}$ and has also been used in other applications, [2]. Indeed more recently the technique has been developed by Laporta in [11] to produce an algorithm which relates all integrals in a Feynman graph to a base set of master integrals. These can then be evaluated by direct methods to complete the overall computation. In the coordinate space representation we use here the basic rule is given in Figure 7

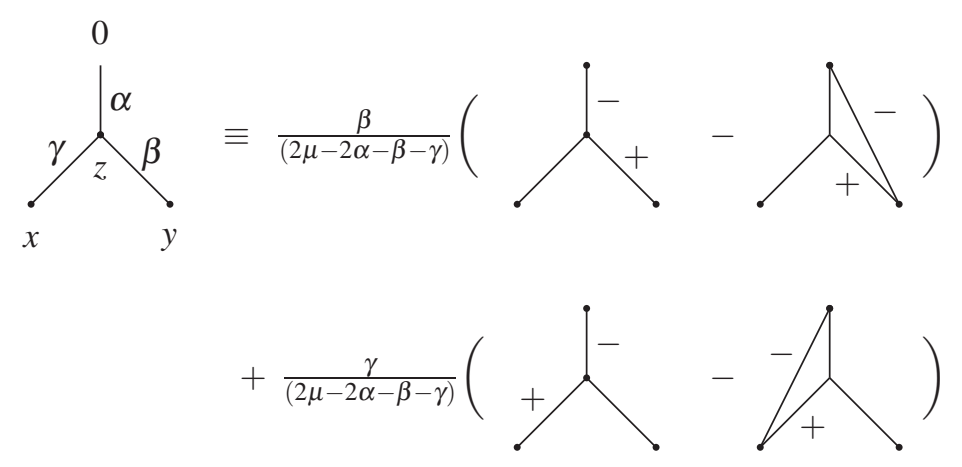

Figure 7. Integration by parts in coordinate space representation. 
where the + or - on a line indicates that the power of that propagator is increased or decreased by unity. For example, with this, [10],

$$
I(1,1,1,1,1)=\frac{v(1,1,2 \mu-2)}{(\mu-2)}[v(1,2,2 \mu-3)-v(3-\mu, 2,3 \mu-5)]
$$

which can be expanded in powers of $\varepsilon$. Clearly the series can only involve rationals and $\zeta_{n}$. Indeed the rule can also be applied to more general cases. In [2] it was shown that

$$
\begin{aligned}
I(\alpha, \mu-1, \mu-1, \beta, \mu-1)=\frac{a(2 \mu-2)}{\Gamma(\mu-1)}[ & \frac{a(\alpha) a(2-\alpha)}{(1-\beta)(\alpha+\beta-2)} \\
& +\frac{a(\alpha+\beta-1) a(3-\alpha-\beta)}{(\alpha-1)(\beta-1)} \\
& \left.+\frac{a(\beta) a(2-\beta)}{(1-\alpha)(\alpha+\beta-2)}\right]
\end{aligned}
$$

for arbitrary $\alpha$ and $\beta$. However, not all graphs can be integrated by parts. An example of such a case is $I(1, \alpha, \beta, \gamma, 1)$ for non-unit $\alpha, \beta$ and $\gamma$. Another example is (15), [7], whose expansion has a non-Riemann zeta value at some point in the expansion. Indeed this is perhaps an indication of an obstruction to integrability.

While integration by parts allows one to reduce the powers of various propagators
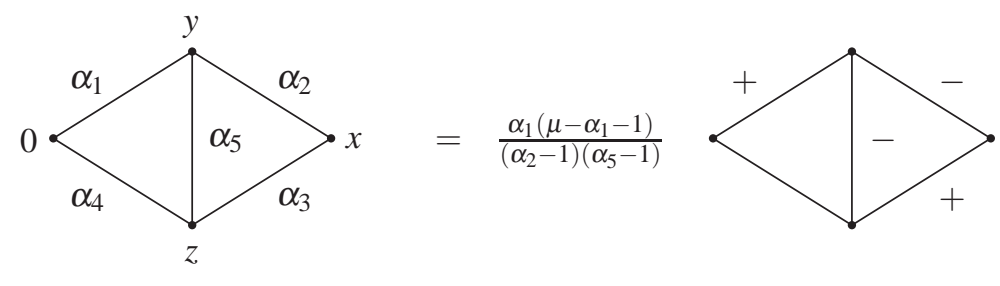

$+\frac{\left(\alpha_{2}+\alpha_{5}-\mu-1\right)}{\left(\alpha_{2}-1\right)}$

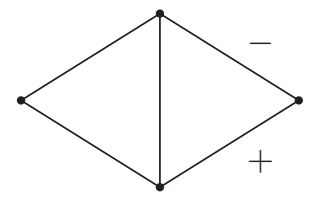

$+\frac{\left(\alpha_{2}+\alpha_{5}-\mu-1\right)}{\left(\alpha_{5}-1\right)}$

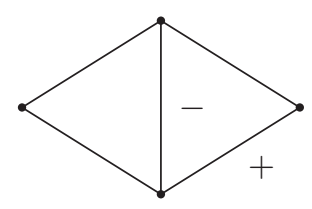

Figure 8. Reduction formula for two loop self energy based on the generalized $\nearrow$ transformation. 
by unity within a Feynman diagram it is not the only method to achieve this. A modification of the uniqueness method can be used to derive rules similar to Figure 7. Specifically if one chooses the exponents of the propagators to be $(2 \mu+1)$ then one finds the extension given in Figure 5. Using this rule and repeating the analysis of the transformations on the two loop self energy graph provides relations specific to this topology, [5]. For instance, extending $\nearrow$ to have the upper vertex exponents summing to $(2 \mu+1)$ gives the relation in Figure 8 where the + or - on the right side indicates that the exponent of that line is increased or decreased by unity. In Figure 8 provided $\alpha_{2} \neq 1$ and $\alpha_{5} \neq 1$ then the powers of the respective propagators can be reduced by unity. However, this restriction is a drawback if one wishes to reduce graphs which have unit exponents. Instead it is possible to extend the method which produced the relation of Figure 8. For instance, rather than begin with the general two loop self energy and applying the generalized uniqueness rule, one can use one of the transformations of [2] and then apply a rule like that of Figure 8 before applying the transformation inverse to the original one. In this way one can build up a suite of relations.

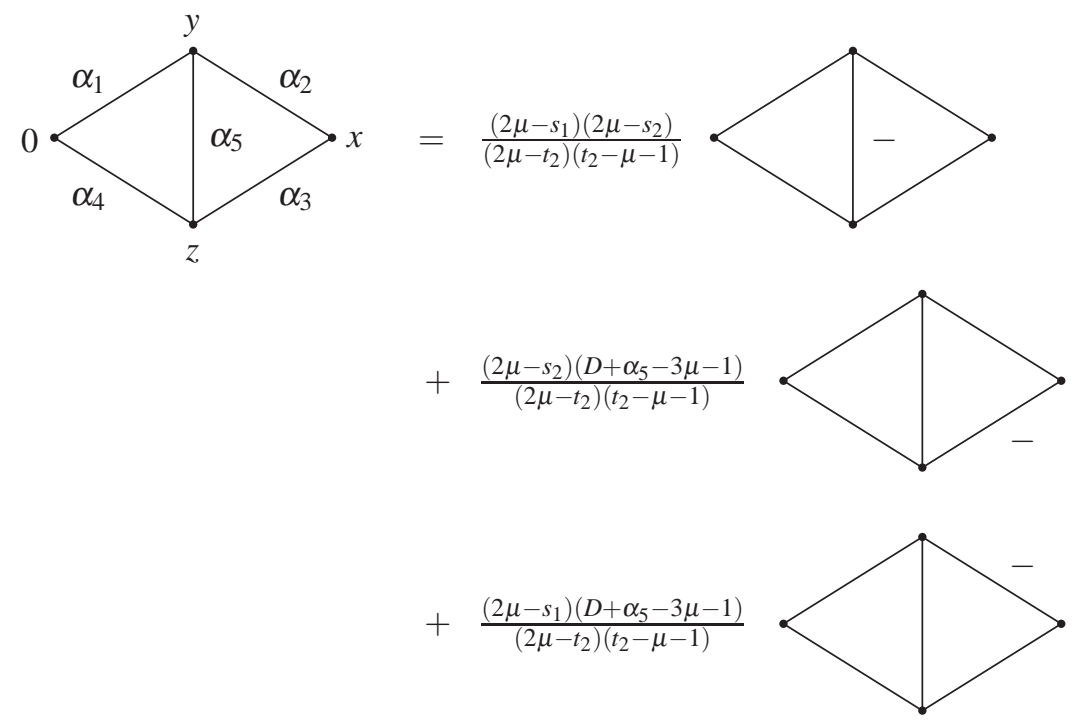

Figure 9. Another reduction formula for two loop self energy.

One such useful relation is illustrated in Figure 9 which is derived in several stages. The first is to construct a relation similar to that of Figure 9 by first applying $\leftarrow$ to the graph of Figure 6 and then undoing it by applying the rule of Figure 5 to the same external vertex. This produces a relation where $t_{2}$ increases by unity in each of the three resulting graphs. The second stage is to apply this rule to the graph of Figure 6 after a CR transformation has been enacted. To complete the derivation the final step is to undo with another CR transformation. Thus the $t_{2}$ value of each graph on the right hand side of Figure 9 is one less than that of the graph on the left 
side. This reduction has coefficients on the right hand side which are non-singular for unit propagators. Other rules can be derived by this method and a fuller set are recorded in Appendix B of [12]. It is worth noting that similar rules based on the generalized uniqueness where developed in [4].

\section{QFT Application}

Having discussed the general techniques for determining massless Feynman integrals using conformal methods, we illustrate their usefulness in a practical problem in a quantum field theory. Specifically we focus on the determination of the critical exponents at a phase transition in various models in the large $N$ expansion. The background which we describe here is based on a series of articles, [1, 2, 13], where exponents were determined in $d$-dimensions at $O\left(1 / N^{2}\right)$ and $O\left(1 / N^{3}\right)$. The fact that $d$-dimensional results are computable means that information on the renormalization group functions can be deduced in various spacetime dimensions. This is due to a special feature of critical point field theories and that is that at a non-trivial fixed point of the renormalization group flow the critical exponents correspond to the associated renormalization group function at that fixed point. Thus information on the renormalization group functions is encoded in these exponents. Moreover, at a fixed point several quantum field theories can lie in the same universality class despite having different structures. This is invariably as a consequence of a common interaction in the Lagrangian. Thus the same exponents can be used to access the structure of the renormalization group functions of two different theories. Further, as the spacetime dimension $d$ is not used as a regulator, information on the exponents can be deduced simultaneously in several different dimensions such as three and four. For more background to the use of the renormalization group equation at near criticality in quantum field theories see, for example, [14].

For the application of the conformal methods we consider here we concentrate on the $O(N)$ nonlinear $\sigma$ model which is critically equivalent in $d$-dimensions to $O(N) \phi^{4}$ theory. For the latter theory the Lagrangian is

$$
L=\frac{1}{2}\left(\partial_{\mu} \phi^{i}\right)^{2}+\frac{g}{8}\left(\phi^{i} \phi^{i}\right)^{2}
$$

where $g$ is the coupling constant and $1 \leq i \leq N$. Introducing an auxiliary field $\sigma$ equates this Lagrangian to

$$
L=\frac{1}{2}\left(\partial_{\mu} \phi^{i}\right)^{2}+\frac{1}{2} \sigma\left(\phi^{i} \phi^{i}\right)-\frac{\sigma^{2}}{2 g} .
$$

At criticality it is the interaction which drives the dynamics and thus it is straightforward to see that in this formulation the Lagrangian interaction is the same as that of the $O(N)$ nonlinear $\sigma$ model when the fields are constrained to lie on an $(N-1)$ dimensional sphere. The constraint would have a final term linear in $\sigma$ rather than a 
quadratic one together with a different coupling constant. This essentially is the origin of both field theories being in the same universality class. The linear or quadratic terms in $\sigma$ at criticality serve effectively to define the structure of the propagators. In coordinate space representation these are, [1, 2],

$$
\left\langle\phi^{i}(0) \phi^{j}(x)\right\rangle=\frac{\delta^{i j} A}{\left(x^{2}\right)^{\alpha}} \quad, \quad\langle\sigma(0) \sigma(x)\rangle=\frac{B}{\left(x^{2}\right)^{\beta}}
$$

where $A$ and $B$ are $x$-independent amplitudes and $\alpha$ and $\beta$ are the scaling dimensions of the fields. The latter comprise two parts. The first is the canonical dimension and the other is the anomalous dimension. Here

$$
\alpha=\mu-1+\frac{1}{2} \eta \quad, \quad \beta=2-\eta-\chi
$$

where $\eta$ is the anomalous dimension of $\phi^{i}$ and $\chi$ is the vertex anomalous dimension. The former is related to the renormalization group function which is also termed the anomalous dimension, $\gamma(g)$, by

$$
\eta=\gamma\left(g_{c}\right)
$$

where $g_{c}$ is the value of the coupling constant at the critical point,

$$
\gamma(g)=\mu \frac{d}{d \mu} \ln Z_{\phi}
$$

and $Z_{\phi}$ is the wave function renormalization constant. (In (31) we have temporarily used $\mu$ to denote the standard renormalization group scale that underlies any renormalization group equation.) To determine the values of the exponents to a particular order in $1 / N$ requires solving the skeleton Schwinger-Dyson equation for the 2point functions at the same order. We do not discuss that formalism here, which can be found in [1, 2], as our focus is rather on the evaluation of the Feynman graphs contributing to these equations. Though we should say that the presence of the nonzero anomalous dimensions in the propagators means that in 2-point functions there are no self energy corrections on any internal propagator as otherwise there would be double counting. So the number of graphs to consider is smaller than the corresponding perturbative case.

The coupling constant at the critical point is denoted by $g_{c}$ and is defined as a nontrivial zero of the $\beta$-function, $\beta\left(g_{c}\right)=0$. As we are working in $d$-dimensions such a non-trivial zero exists in our theories since away from the spacetime dimension where the theory is renormalizable the coupling constant becomes dimensionful. Hence the first term of the $d$-dimensional $\beta$-function depends on $d$. Moreover, $g_{c}$ will depend on the parameters of the theory which in our case here is $N$. Thus $g_{c}=g_{c}(d, N)$. Similarly $\eta=\eta(d, N)$ and $\chi=\chi(d, N)$. These can all be expanded in powers of $1 / N$ where $N$ is large in such a way that the coefficients of $1 / N$ are $d$-dependent. Thence if one expresses these coefficients in powers of $\varepsilon$ where $d=4-2 \varepsilon$ for $\phi^{4}$ theory or $d=2+\bar{\varepsilon}$ for the nonlinear $\sigma$ model, then one can 
deduce the coefficients in the corresponding renormalization group equation to all orders in perturbation theory at that order in $1 / N$. In this respect it is important to note that in the large $N$ expansion $\varepsilon$ or $\bar{\varepsilon}$ do not play the role of a regulator as they would do in conventional perturbation theory.

Instead to see the origin of where a regulator is required one should consider the simple two loop contribution to the $\sigma$ self energy graph given in Figure 10 in coordinate space representation.

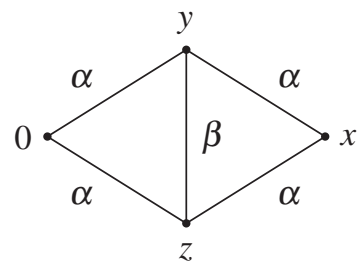

Figure 10. Two loop self energy for $\sigma$.

To use conformal methods one has to check the sum of the exponents at a vertex in coordinate space representation. From (29) one can see that

$$
2 \alpha+\beta=2 \mu-\chi .
$$

However, from the structure of the renormalization group equation at criticality the anomalous dimensions $\eta$ and $\chi$ begin as $O(1 / N)$. More, specifically

$$
\eta=\sum_{i=1}^{\infty} \frac{\eta_{i}}{N^{i}}
$$

Thus at leading order in $1 / N$ the basic vertex is unique, [2]. Hence at this order one can integrate at either of the vertices and produce the first contribution to the integral which is $v(\mu-1, \mu-1,2)$. The second integration is a simple chain and naively gives $v(\mu, \mu, 0)$. This is clearly ill-defined due to the zeroes and singularities deriving from the $\Gamma$-function. However, this graph was chosen to illustrate the fact that the graph and indeed the theory requires a regularization in this critical point formulation. The method developed in [1, 2] was to use analytic regularization which is introduced by shifting the vertex anomalous dimension by an infinitesimal amount, $\Delta$, via

$$
\beta \rightarrow \beta-\Delta .
$$

In some respect one is in effect performing a perturbative expansion in the vertex anomalous dimension, [1, 2]. Consequently even at leading order the graph of Figure 10 no longer has a unique vertex due to a non-zero $\Delta$. Therefore, to determine the graph to the finite part in $\Delta$ requires the addition and subtraction of the graphs of Figure 11, [2]. 

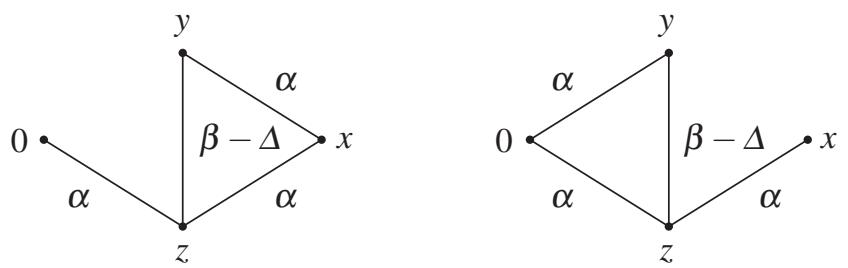

Figure 11. Subtracted graphs for computation of $\sigma$ two loop self energy.

These two graphs have been chosen in such a way that their singularity structure in $\Delta$ exactly matches that of Figure 10, [2]. Clearly they represent simple chain integrals which can be determined as $2 v(\alpha, \beta-\Delta, 2 \mu-\alpha-\beta+\Delta) v(\alpha, \mu-\Delta, \mu-\alpha+\Delta)$ where the singularity is clearly regularized. To complete the evaluation introduces another technique, which we will use later, to extract a finite term of a graph. This is a temporary regularization, [2]. If one subtracts the graphs of Figure 11 from that of Figure 10, the combination is finite with respect to $\Delta$ which is therefore not required and can be set to zero. Thus one can complete the first integration at the upper vertex of each graph. (Without a regularization the point where one integrates in each graph has to be the same and thence the order of integration is important.) This produces $v(\alpha, \alpha, \beta)$ for each graph. However, each of the three subsequent chain integrals has a singular exponent, $\mu$. To circumvent this the lower two propagators of all three graphs are temporarily regularized by $\alpha \rightarrow \alpha-\delta$ where $\delta$ is arbitrary. Thus the three graphs give

$$
\begin{aligned}
& {[v(\mu-\delta, \mu-\delta, 2 \delta)-v(\alpha-\delta, \mu-\delta, \mu-\alpha+2 \delta)} \\
& -v(\mu-\delta, \alpha-\delta, \mu-\alpha+2 \delta)] v(\alpha, \alpha, \beta)
\end{aligned}
$$

which is clearly finite as $\delta \rightarrow 0$, [2]. Thus to $O(\Delta)$ the graph of Figure 10 evaluates to, [2],

$$
\frac{2 \pi^{2 \mu} a^{2}(\alpha) a(\beta)}{\Gamma(\mu)}\left[\frac{1}{\Delta}+B(\beta)-B(\alpha)+O(\Delta)\right]
$$

where $B(z)=\psi(z)+\psi(\mu-z)$ for $z$ and $(\mu-z)$ not equal to zero or a negative integer and $\psi(z)$ is derivative of the logarithm of the $\Gamma$-function.

A more involved example which uses many of the techniques of the previous section occurs in the computation of the $O\left(1 / N^{2}\right)$ correction to the $\beta$-function in $O(N) \phi^{4}$ theory. The relevant critical exponent is $\omega$ which is related through the critical renormalization group equation to the $\beta$-function slope at criticality. In this case it has the form

$$
\omega=2-\mu+\sum_{n=1}^{\infty} \frac{\omega_{n}}{N^{n}}
$$

and the explicit forms for $\omega_{n}$ are deduced from the part of the Schwinger-Dyson equations corresponding to corrections to scaling. In other words the propagators of 
(28) are extended to

$$
\begin{aligned}
\left\langle\phi^{i}(0) \phi^{j}(x)\right\rangle & =\frac{\delta^{i j} A}{\left(x^{2}\right)^{\alpha}}\left[1+A^{\prime}\left(x^{2}\right)^{\omega}\right] \\
\langle\sigma(0) \sigma(x)\rangle & =\frac{B}{\left(x^{2}\right)^{\beta}}\left[1+B^{\prime}\left(x^{2}\right)^{\omega}\right] .
\end{aligned}
$$

In principle other corrections can appear here corresponding to other exponents such as that for the $\beta$-function of the nonlinear $\sigma$ model but one tends to focus on one calculation at a time. The effect of the corrections is that to deduce $\omega_{n}$ within the Schwinger-Dyson formalism all Feynman diagrams with one correction insertion on a propagator contribute at each particular order in $1 / N$. While the $O\left(1 / N^{2}\right)$ expression for $\omega$ appeared in [15] the explicit evaluation of the contributing graphs has not been detailed. Thus we discuss one such diagram here as the approach can be readily adapted to the other graphs. It is given in Figure 12. To see that it is $O\left(1 / N^{2}\right)$ each closed loop of $\phi^{i}$ fields contributes a factor of $N$ and each $\sigma$ propagator is $O(1 / N)$. This is due to the fact that the amplitude $B$ is $O(1 / N)$, [1, 2]. As there are four of the former and five of the latter then this gives $O(1 / N)$ overall which is one factor of $1 / N$ more than the previous order graph of Figure 10. Finally, another factor of $1 / N$ derives from the actual Schwinger Dyson formalism used to determine $\omega_{2}$. The double line on one $\sigma$ propagator in Figure 12 denotes the $B^{\prime}$ correction.

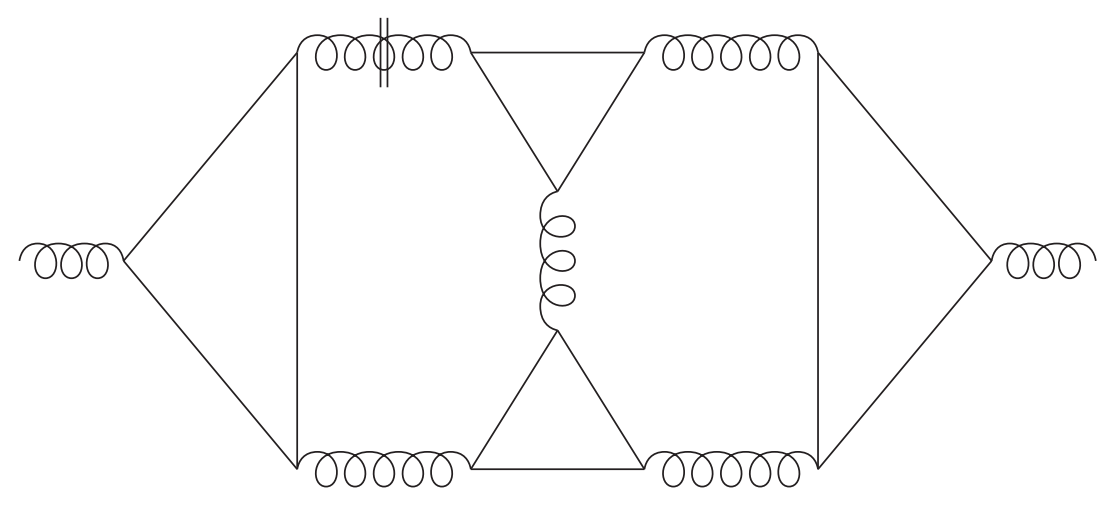

Figure 12. Particular graph contributing to the $\phi^{4}$ theory $\beta$-function at $O\left(1 / N^{2}\right)$.

The presence of such a correction means that the graph is $\Delta$-finite. Moreover, since we only want the value as a function of $d$ rather than $d$ and $N$ we can replace the exponents of the lines by their canonical values. If one was computing $\omega_{3}$ then the anomalous dimensions of each exponent would need to be retained at $O(1 / N)$. The benefit of this restriction here is that of the ten vertices eight are unique. There are ten integrations to do over the vertices rather than the six of the loops as we are in 
coordinate space representation. Given this high degree of uniqueness the graph can be reduced rather quickly to one with fewer integrations. To do this one can use a variety of the rules we had earlier aside from uniqueness such as conformal transformation, unique triangle, insertion at an internal or external vertex. Ultimately one produces the graph of Figure 13.

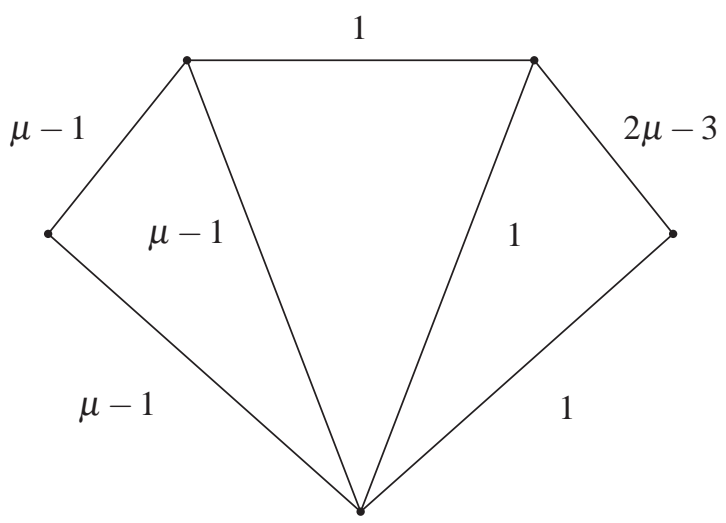

Figure 13. Reduced integral of Figure 12.

This graph cannot be reduced any further since there are no unique vertices or triangles. Though various vertices or triangles are one unit from uniqueness. Moreover, integration by parts cannot be used since at some point one produces an unregularized exponent, such as 0 or $\mu$, or a zero in a denominator factor. In some sense this graph could be regarded as a master integral since it arises in several of the other graphs contributing to the $\sigma$ Schwinger-Dyson equation. Moreover, it is worth noting that in strictly four dimensions the propagators of the graph would all have unit exponents. As an aside if an interested reader has been applying the conformal techniques to reduce the diagram and obtains similar exponents but distributed differently around the diagram then it will be related to that of Figure 13 by applying the transformations discussed for the master 2-loop self energy. We note here that if a conformal transformation is applied to the graph of Figure 13 with the left internal point as the CL base, then that would introduce a new line from the top right internal vertex to the base. This illustrates comments made earlier.

To proceed further and reduce the graph to a known function of $d$ requires an integration by parts but this requires modifying the integral first. Though before this can be achieved safely one has to introduce a temporary regularization to handle hidden singularities at a later stage of the computation. This technique has been applied by others, [4, 16]. For our case we have choosen the regularization of Figure 14. How one chooses the temporary regularization is not unique. However, it is chosen here so that after application of the integration by parts rule of Figure 7 the resulting four graphs have either unique vertices or triangles which are $\delta$-dependent 
and which regularize any singularity after subsequent integration. For the integration by parts we use the top left

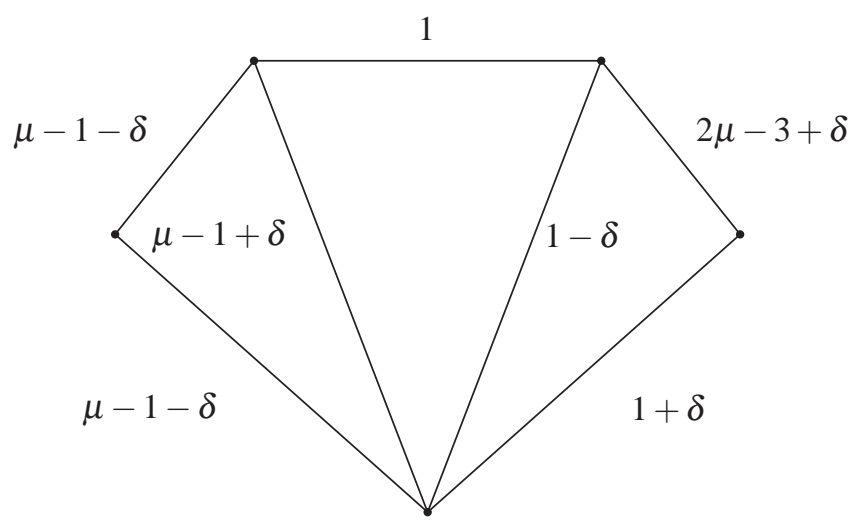

Figure 14. Temporary regularization of previous graph to reduce it to two loop basic graphs.

internal vertex of Figure 14 with the line joining the quartic vertex as the reference line of the rule of Figure 7. This produces the four graphs of Figures 15-18.

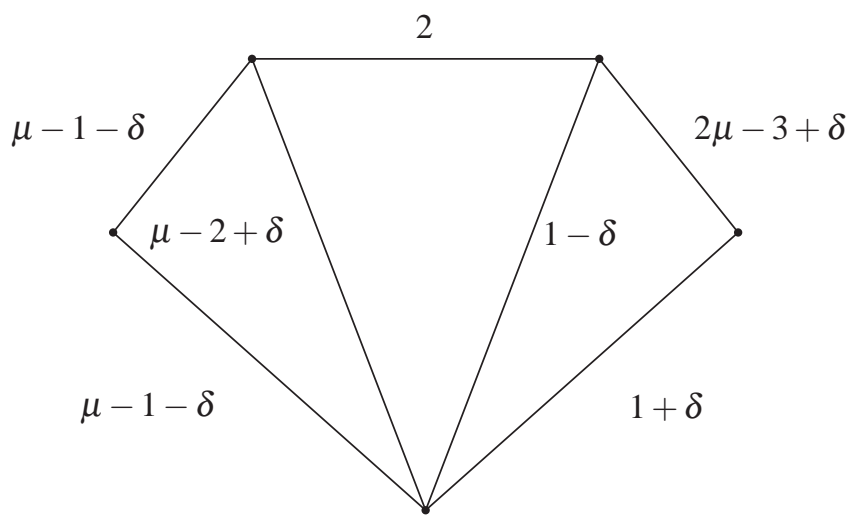

Figure 15. First graph after integration by parts.

All but the third have at least one unique vertex while that has a unique triangle. In our earlier notation the first two graphs of Figure 15 and 16 are

$$
v(2,1-\delta, 2 \mu-3+\delta) I(\mu-1-\delta, \mu-1+\delta, \mu-1+\delta, \mu-1-\delta, 1)
$$

and

$$
v(2, \mu-1-\delta, \mu-1+\delta) I(1+\delta, 2 \mu-3,1+\delta, 2 \mu-3-\delta, 1) .
$$


As both of these are $\delta$-finite and have no $\delta$-singular coefficients, one can set $\delta$ to zero in each. The final evaluation is by a two loop reduction formula similar to those of Figures 8 and 9.

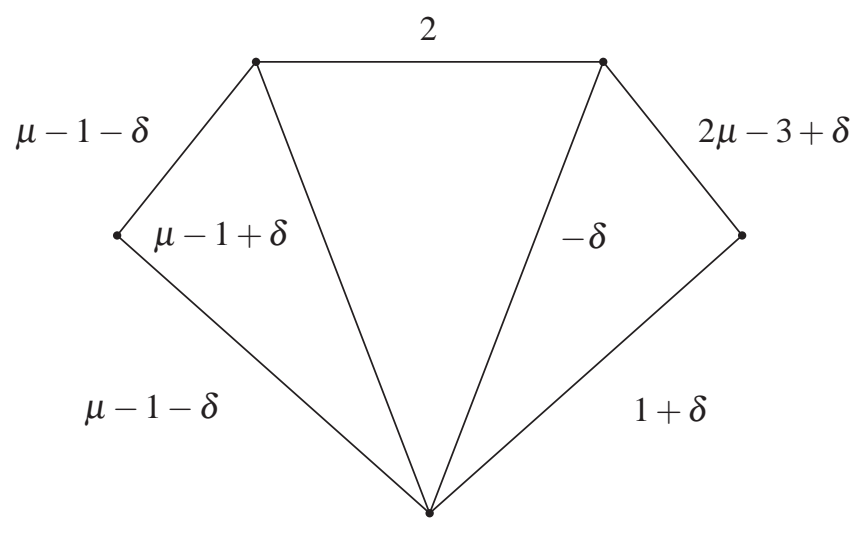

Figure 16. Second graph after integration by parts.

For the remaining two graphs of Figures 17 and 18 one has to treat them together due to the singular propagator exponents as will be evident. After integrating the respective unique triangle and vertex they combine to produce

$$
\begin{array}{r}
a^{3}(1) a(\mu-\delta) a(2 \mu-3+\delta)[I(\mu-1, \mu-1,1+\delta, \mu-1-\delta, \mu-1 \\
-I(\mu-1, \mu-1,1+\delta, \mu-1-2 \delta, \mu-1+\delta)]
\end{array}
$$

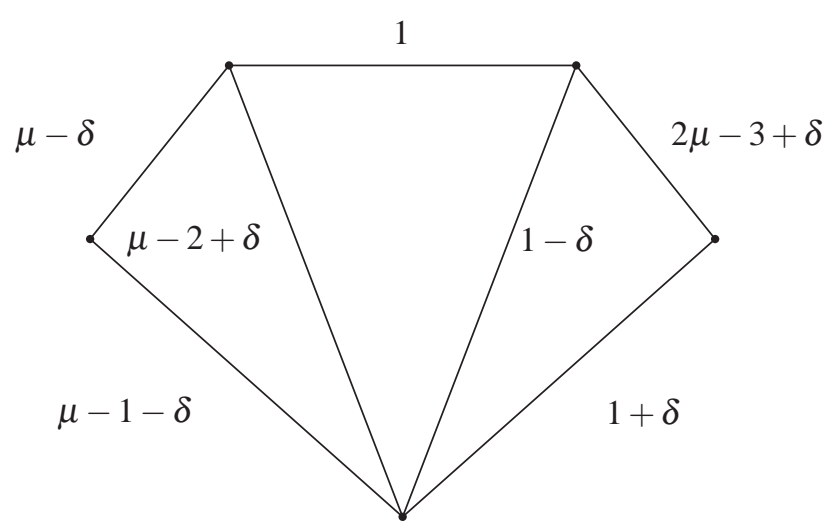

Figure 17. Third graph after integration by parts.

As the external coefficient includes a factor of $1 / \delta$ then the quantity inside the square brackets needs to be evaluated to $O(\delta)$. 


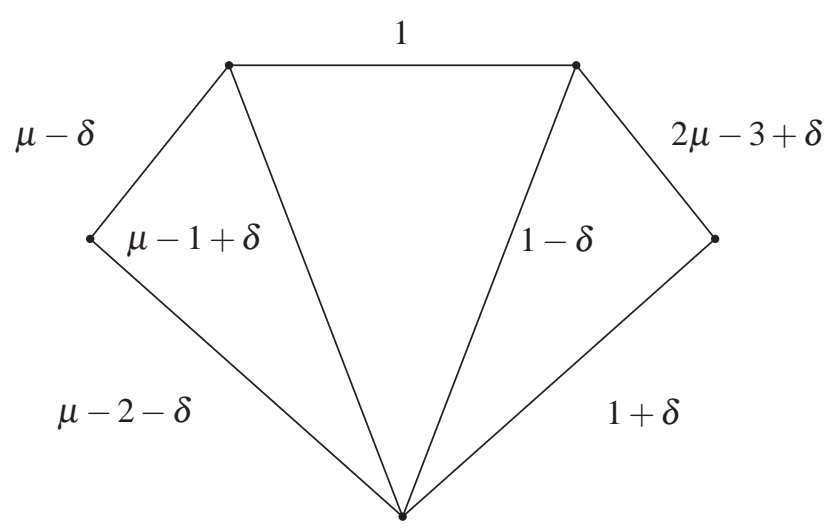

Figure 18. Fourth graph after integration by parts.

This is not possible exactly for both integrals. (It is for the first.) Instead since one only needs the $O(\delta)$ part itself one can achieve this by evaluating the integral

$$
I(\mu-1, \mu-1,1, \mu-1-\delta, \mu-1+\delta) .
$$

From the two 2-loop graphs we are interested in the $O(\delta)$ term of this integral clearly corresponds to the piece we require. Moreover, it can be evaluated exactly using $\downarrow$ as it then reduces to an integral to which one can apply a 2-loop recurrence relation similar to that of Figure 9. The final expression for the graph of Figure 12 is

$$
\frac{a(\mu-1) a^{2}(2 \mu-3) a(2 \mu-2)}{2(\mu-3)(\mu-2)^{9}}\left[f_{2}-f_{1}^{2}-\frac{2 f_{1}}{(\mu-2)}+6 f_{3}\right]
$$

where

$$
\begin{aligned}
& f_{1}=\psi(3-\mu)+\psi(2 \mu-3)-\psi(\mu-1)-\psi(1) \\
& f_{2}=\psi^{\prime}(3-\mu)-\psi^{\prime}(2 \mu-3)+\psi^{\prime}(\mu-1)-\psi^{\prime}(1) \\
& f_{3}=\psi^{\prime}(\mu-1)-\psi^{\prime}(1) .
\end{aligned}
$$

Setting $\mu=2$ reproduces the established leading order value for the wheel of three spokes, [17], which provides a useful check. Finally, all the other contributing graphs are evaluated in a similar way and the full expression for $\omega_{2}$, after using the Schwinger Dyson formalism, is given in [15].

\section{Future Directions}

We close the article by discussing several directions in which this approach could move. First, the extension of scalar field theories to non-abelian gauge theories has 
been considered in [18, 19, 20, 21, 22] for various applications where information is needed on the renormalization group functions of operators in deep inelastic scattering and the $\beta$-function. That approach is based on the observations of [23] using the number of quark flavours, $N_{f}$, as the expansion parameter. Rather than use the full QCD Lagrangian one exploits the critical point equivalence with the non-abelian Thirring model, [23],

$$
L=i \bar{\psi}^{i} \not \partial \psi^{i}-\frac{1}{2}\left(A_{\mu}^{a}\right)^{2}
$$

where $D_{\mu}$ is the covariant derivative, $T^{a}$ are the group generators and $\psi^{i}$ is the quark field with $1 \leq i \leq N_{f}$. The spin-1 auxiliary field $A_{\mu}^{a}$ plays the role of the gluon in the higher dimensional theory. The triple and quartic gluon vertices of QCD are generated by the 3-point and 4-point functions of (45) with $A_{\mu}^{a}$ external legs respectively. Following the critical point analysis the propagators in a similar notation, but in momentum space, are

$$
\begin{aligned}
\left\langle\psi^{i}(-p) \psi^{j}(p)\right\rangle & =\frac{\delta^{i j} A \not p}{\left(p^{2}\right)^{\mu-\alpha}} \\
\left\langle A_{\mu}^{a}(-p) A_{v}^{b}(p)\right\rangle & =-\frac{\delta^{a b} B}{\left(p^{2}\right)^{\mu-\beta}}\left[\eta_{\mu v}-\xi \frac{p_{\mu} p_{v}}{p^{2}}\right]
\end{aligned}
$$

where $\xi$ is the gauge parameter with the Landau gauge corresponding to $\xi=1$. From dimensional analysis the exponents are now, [18],

$$
\alpha=\mu+\frac{1}{2} \eta \quad, \quad \beta=1-\eta-\chi
$$

which means the basic vertex is one step from uniqueness. This complicates computations in that to proceed one has to break all contributing graphs into scalar integrals and treat them by transformations, subtractions or use integration by parts to reduce them to computable cases. While it has been possible to do this in certain instances, [19, 20, 22], it is not systematic.

Since the application of the method of [1, 2] to QCD an algorithm has been developed which allows one to exploit integration by parts. Known as the Laporta algorithm, [11], it creates all integration by parts relations between integrals of a particular topology and then algebraically solves them in such a way that all integrals are reduced to a basis set of master integrals. Once their values are known by other methods then the problem is complete. In the large $N_{f}$ context once one moves to say $O\left(1 / N_{f}^{2}\right)$ computations then graphs such as that of Figure 12 need to be computed in QCD. Then the solid lines would represent quarks and the springs would correspond to gluons. However, taking the traces over the closed loops results in a huge number of irreducible numerator scalar products. While the propagators do not have integer powers, as is the case in perturbative calculations, there appears to be a similarity to the problem. In other words in principle a generalization of the Laporta algorithm should be able to produce a reduction of the irreducible graphs to a set of masters. The difficulty is that the presence of non-integer propagator powers means that the present Laporta algorithm would need to be modified in order to have a point, akin to a ground state, below which no more reductions could be possible. 
Though it is not clear under what conditions such a bottom point exists or whether for certain topologies or distribution of non-unit exponents it can be proved to be impossible. Indeed the latter point could be related to the issue of lack computability of a graph due to the presence of multiple-zeta values similar to (15). However, it seems that for the practical problem of deducing the QCD $\beta$-function at $O\left(1 / N_{f}^{2}\right)$ such an extension to the Laporta algorithm is possibly the only feasible tactic at present.

Aside from this possible extension to the Laporta algorithm another interesting possibility is to what extent the conformal integration methods can be built into that algorithm to improve and speed reductions within a computer algebra programme for massless Feynman graphs. This may be important for higher loop topologies. For instance, earlier we derived recurrence relations for the two loop self energy topology based on the transformation deduced from the generalized uniqueness condition. While such relations are no doubt contained within integration by parts relations of the Laporta construction, that of Figure 9 is particularly useful in that there is no increase in the power of any propagator. Therefore, it may be possible to construct similar relations using conformal transformations but for higher loop massless topologies. Indeed such transformations are not unrelated to the symmetry group of the topology as has already been studied in depth for the two loop self energy, [6, 7]. At the time of [6, 7] expanding a graph in terms of its group invariants was a promising approach which was complemented by later methods such as [8, 9]. However, it may be worth returning to a group theory analysis for topologies such as that represented in Figure 13. This is because the high order expansion in terms of $\varepsilon$ of this and other three and four loop topologies will soon be required for extending QCD to five and possibly higher loops. In this respect another direction of exploration may be to study the structure of the graph polynomials of a topology. The transformations of [2] have been derived from a graphical approach to understanding the structure of the two loop self energy graph. Understanding the effect such conformal transformations have on the graph polynomials of massless integrals may also give insight into the as yet undetermined group theory properties of higher order topologies.

Acknowledgements It is with pleasure that I thank the organizers of the meeting for permission to include this article in these proceedings. It is based on a talk presented at Quantum Field Theory, Periods and Polylogarithms III, Humboldt University, Berlin in June 2012 which was also in

honour of Dr D.J. Broadhurst's 65th birthday. The AXODRAW package, [24], was used to draw the figures in the article.

\section{References}

1. Vasil'ev, A.N., Pismak, Y.M., Honkonen, J.R.: Simple method of calculating the critical indices in the $1 / N$ expansion. Theor. Math. Phys. 46, 104-113 (1981)

2. Vasil'ev, A.N., Pismak, Y.M., Honkonen, J.R.: $1 / N$ expansion: calculation of the exponents $\eta$ and $v$ in the order $1 / N^{2}$ for arbitrary number of dimensions. Theor. Math. Phys. 47, 465-475 
(1981)

3. d'Eramo, M., Peliti, L., Parisi, G.: Theoretical predictions for critical exponents at the $\lambda$-point of Bose liquids. Lett. Nuovo Cim. 2, 878-880 (1971)

4. Ussyukina, N.I.: Calculation of multiloop diagrams in high orders of perturbation theory. Phys. Lett. B267, 382-388 (1991)

5. Gracey, J.A.: On the evaluation of massless Feynman diagrams by the method of uniqueness JAG. Phys. Lett. B277, 469-473 (1992)

6. Broadhurst, D.J.: Exploiting the 1,440-fold symmetry of the master two-loop diagram. Z. Phys. C32, 249-253 (1986)

7. Barfoot, D.T., Broadhurst, D.J.: $Z_{2} \times S_{6}$ symmetry of the two-loop diagram. Z. Phys. C41. 81-85 (1988)

8. Broadhurst, D.J., Kreimer, D.: Knots and numbers in $\phi^{4}$ theory to 7 loops and beyond. Int. J. Mod. Phys. C6, 519-524 (1995)

9. Bierenbaum, I., Weinzierl, S.: The massless two loop two point function. Eur. Phys. J. C32, 67-78 (2003)

10. Chetyrkin, K.G., Tkachov, F.V.: Integration by parts: the algorithm to calculate $\beta$-functions in 4 loops. Nucl. Phys. B192, 159-204 (1981)

11. Laporta, S.: High precision calculation of multiloop Feynman integrals by difference equations. Int. J. Mod. Phys. A15, 5087-5159 (2000)

12. Gracey, J.A.: Computation of critical exponent $\eta$ at $O\left(1 / N_{f}^{2}\right)$ in quantum electrodynamics in arbitrary dimensions. Nucl. Phys. B414, 614-648 (1994)

13. Vasil'ev, A.N., Pismak, Y.M., Honkonen, J.R.: $1 / N$ expansion: calculation of the exponent $\eta$ in the order $1 / N^{3}$ by the conformal bootstrap method. Theor. Math. Phys. 50, 127-134 (1982)

14. Zinn-Justin, J.: Quantum field theory and critical phenomena, International Series of Monographs on Physics. Clarendon Press, Oxford (2002)

15. Broadhurst, D.J., Gracey, J.A., Kreimer, D.: Beyond the triangle and uniqueness relations: non-zeta counterterms at large $N$ from positive knots. Z. Phys. C75, 559-574 (1997)

16. Ussyukina, N.I.\& Davydychev, A.I.: New results for two-loop off-shell three-point diagrams. Phys. Lett. B332, 159-167 (1994)

17. Broadhurst, D.J.: Evaluation of a class of Feynman diagrams for all numbers of loops and dimensions. Phys. Lett. B164, 356-360 (1985)

18. Gracey, J.A.: Quark, gluon and ghost anomalous dimensions at $O\left(1 / N_{f}\right)$ in quantum chromodynamics. Phys. Lett. B318, 177-183 (1993)

19. Gracey, J.A.: The QCD $\beta$-function at $O\left(1 / N_{f}\right)$. Phys. Lett. B373, 178-184 (1996)

20. Ciuchini, M., Derkachov, S.E., Gracey, J.A., Manashov, A.N.: Computation of quark mass anomalous dimension at $O\left(1 / N_{f}^{2}\right)$ in quantum chromodynamics. Nucl. Phys. B579, 56-100 (2000)

21. Gracey, J.A.: Anomalous dimension of non-singlet Wilson operators at $O\left(1 / N_{f}\right)$ in deep inelastic scattering. Phys. Lett. B322, 141-146 (1994)

22. Bennett, J.F., Gracey, J.A.: Determination of the anomalous dimension of gluonic operators in deep inelastic scattering at $O\left(1 / N_{f}\right)$. Nucl. Phys. B517, 241-268 (1998)

23. Hasenfratz, A., Hasenfratz, P.: The equivalence of the $S U(N)$ Yang-Mills theory with a purely fermionic model. Phys. Lett. B297, 166-170 (1992)

24. Vermaseren, J.A.M.: Axodraw. Comput. Phys. Commun. 83, 45-58 (1994) 